\title{
¿Dentro o fuera del clóset? Apertura y/o encubrimiento de la orientación sexual de estudiantes de Medicina de la Universidad Nacional de Asunción
}

\author{
In or out of the closet? Disclosure and/or concealment of the sexual orientation \\ of medical students of the National University of Asunción
}

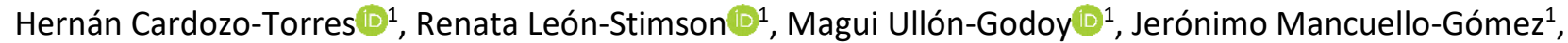 \\ Camila Palma-Cegla $\bigotimes^{1}$, Tudor Pipirig ${ }^{2}$, Leonardo José Consoni ${ }^{3}$, Sandra González-Almada ${ }^{(1}{ }^{1}$, Julio Torales $\oplus^{1}$
}

${ }^{1}$ Universidad Nacional de Asunción, Facultad de Ciencias Médicas, San Lorenzo, Paraguay.

${ }^{2}$ Carol Davila University of Medicine and Pharmacy, Romania

${ }^{3}$ Universidad del Sur de Santa Catarina, Brasil

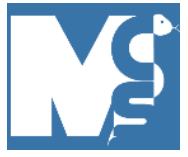

Recibido: 07/11/2020

Revisado: 07/11/2020

Aceptado:01/12/2020

\section{Autor correspondiente}

Julio Torales

Universidad Nacional de Asunción

San Lorenzo - Paraguay

jtorales@med.una.py

\section{Conflictos de interés}

Los autores declaran no poseer conflictos de interés.

\section{Fuente de financiación}

Los autores no recibieron apoyo financiero para la investigación, autoría y/o publicación de este artículo.

Este artículo es publicado bajo una licencia de Creative Commons Reconocimiento 4.0 Internacional.

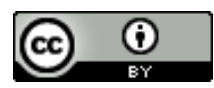

\section{RESUMEN}

Introducción: la orientación sexual es la atracción emocional, afectiva, romántica o sexual que uno siente hacia los demás. Su apertura y encubrimiento son variables que han sido ampliamente relacionadas con el estado de salud mental. Metodología: estudio observacional descriptivo, de corte transversal y temporalmente prospectivo, con muestreo no probabilístico, que incluyó a 277 estudiantes de Medicina (campus de Sajonia y de San Lorenzo, y Filial Santa Rosa del Aguaray) de la Facultad de Ciencias Médicas de la Universidad Nacional de Asunción. La apertura/encubrimiento de la orientación sexual se determinó a través de adaptaciones españolas del "Outness Inventory" y de la "Sexual Orientation Concealment Scale". Resultados: las escalas utilizadas mostraron buenas consistencias internas (alpha de Cronbach=0,90). 17,3\% de los encuestados se definía como parte de las minorías sexuales. Se encontraron diferencias estadísticamente significativas en cuanto a los niveles de apertura de la orientación sexual en personas heterosexuales y en personas $\mathrm{LGB}+[\mathrm{t}(275)=8,2, \mathrm{p}<0,01]$. En la población de estudiantes de Medicina $\mathrm{LGB}+$, se observaron menores niveles de apertura primero hacia la religión, y luego hacia la familia, Facultad y amigos $(p<0,01)$. El $75 \%$ de los estudiantes de Medicina LGB+ ocultaron de alguna forma su orientación sexual en las últimas dos semanas. Conclusión: la Facultad de Ciencias Médicas de la Universidad Nacional de Asunción debe considerar examinar de cerca su ambiente y cultura organizacional, así como su currículo, con el fin de garantizar que exista un entorno positivo e inclusivo para todos los estudiantes. La adopción de la diversidad en la educación médica permite formar médicos que puedan practicar la Medicina de manera competente, compasiva y con enfoque de derechos humanos.

Palabras clave: Orientación sexual; apertura; ocultamiento; minorías sexuales; estudiantes de medicina

\section{ABSTRACT}

Introduction: Sexual orientation is the emotional, affective, romantic or sexual attraction that one feels towards others. Its disclosure and concealment are variables that have been widely related to mental health status. Methodology: This was a descriptive, cross-sectional and temporally prospective observational study, with a non-probabilistic sample, which included 277 medical students (Sajonia, San Lorenzo, and Santa Rosa del Aguaray campuses) from the Faculty of Medical Sciences of the National University of Asunción. The disclosure/concealment of sexual orientation was determined through Spanish adaptations of the "Outness Inventory" and the "Sexual Orientation Concealment Scale". Results: The scales used showed good internal consistencies (Cronbach's $\alpha=0.90$ ). 17.3\% of those surveyed defined themselves as part of sexual minorities. Statistically significant differences were found in the levels of disclosure of sexual orientation in heterosexual people and in LGB+ people $[\mathrm{t}(275)=8.2, \mathrm{p}<0.01]$. In the population of $\mathrm{LGB}+$ medical students, lower levels of disclosure were observed, first towards religion, and then towards family, Faculty, and friends $(p<0.01) .75 \%$ of LGB+ medical students concealed their sexual orientation in some way in the past two weeks. Conclusion: The Faculty of Medical Sciences of the National University of Asunción should consider closely examining its environment and organizational culture, as well as its curriculum, in order to guarantee that there is a positive and inclusive environment for all students. The adoption of diversity in medical education enables the training of physicians who can practice medicine competently, compassionately, and with a human rights approach. .

Keywords: Sexual orientation; disclosure; concealment; sexual minorities; medical students.

Cómo citar este artículo: Cardozo-Torres H, Leon-Stimson R, Ullón-Godoy M, Mancuello-Gómez J, Palma-Cegla C, Pipirig T, et al. ¿Dentro o fuera del clóset? Apertura y/o encubrimiento de la orientación sexual de estudiantes de Medicina de la Universidad Nacional de Asunción. Med. clín. soc. $2021 ; 5(1): 3-10$ 


\section{INTRODUCCIÓN}

Investigaciones anteriores han indicado que los estudiantes que se identifican como lesbianas, gais, bisexuales o de otras minorías sexuales ( $L G B+$ ) a menudo tienen experiencias negativas en sus Facultades debido a su orientación sexual o identidad de género (1). La orientación sexual se puede definir como la atracción emocional, afectiva, romántica o sexual que se siente hacia otros. La orientación sexual es duradera y va desde la heterosexualidad completa hasta la homosexualidad completa, incluyendo varias formas de bisexualidad (2).

Una investigación realizada en Estados Unidos de América y en Canadá examinó la orientación sexual de estudiantes de Medicina y si se estos se encontraban o no "fuera del clóset" en la Universidad. El estudio reportó que los estudiantes ocultaban su orientación sexual a lo largo de su formación médica por diversas razones, entre las que destacaban el miedo a la discriminación y la necesidad de ajustarse a las normas sociales y culturales imperantes (3). En América Latina, específicamente en Perú, se entrevistó a hombres homosexuales sobre el proceso de aceptación de su orientación sexual y de "salida del clóset". Los participantes mencionaron haber experimentado sentimientos de negación en relación con sus propias atracciones $y$, en algún momento, llegaron a estar confundidos y a considerarse diferentes (4). En Paraguay, por su parte, investigadores abordaron la discriminación que existe hacia personas con orientación sexual diferente a la heterosexual en el ambiente laboral, encontrándose que las mujeres tenían una actitud más favorable hacia personas homosexuales en comparación con los hombres, con niveles de homofobia más elevados en estos últimos (5).

Según la Real Academia de la Lengua Española, la palabra fobia se define como un "temor angustioso e incontrolable ante ciertos actos, ideas, objetos o situaciones, que se sabe absurdo y se aproxima a la obsesión" (6). Sin embargo, en el caso específico de la homofobia, su conceptualización ha ido evolucionando en los últimos años (7) y se sostiene en una serie de niveles. Estos niveles se resumen en la tabla 1 (8).

TABLA 1. NIVELES DE HOMOFOBIA.

\begin{tabular}{cl}
\hline Nivel & \multicolumn{1}{c}{ Características } \\
\hline \multirow{2}{*}{ Personal } & $\begin{array}{l}\text { Se asienta sobre un sistema personal de creencias, tales como sentir compasión por la } \\
\text { "incapacidad de los homosexuales de controlar sus deseos", odio por considerarlos } \\
\text { psicológicamente trastornados, genéticamente defectuosos o inadaptados, cuya existencia } \\
\text { contradice las leyes de la naturaleza, espiritualmente inmorales, infectados, "asquerosos" o } \\
\text { inferiores a los heterosexuales. }\end{array}$ \\
& $\begin{array}{l}\text { En este nivel, la homofobia afecta las relaciones entre los individuos, por ejemplo, poner } \\
\text { apodos o hacer chistes, agredir física o verbalmente, retirar apoyos y rechazar a la persona. }\end{array}$ \\
Interpersonal & Se refiere a las formas en las que organismos gubernamentales, educativos o religiosos \\
Institucional & discriminan sistemáticamente, por ejemplo, a través de leyes y su aplicación. \\
& Nivel que engloba normas sociales o códigos de conducta que, sin estar expresamente \\
inscritos en una ley o reglamento, funcionan en la sociedad para legitimar la opresión.
\end{tabular}

A muchos estudiantes LGB+ les resulta difícil concentrarse en lo académico debido a la estigmatización, la intolerancia, el estrés crónico y la discriminación (9). Para los estudiantes de Medicina, el éxito académico es crucial en la generación de una base para la atención eficaz al paciente, y la evidencia sugiere que los estudiantes de Medicina LGB+ experimentan una serie de barreras a medida que avanzan en el sistema de educación médica (10).

Tomando en cuenta todo lo anterior, el propósito de este estudio fue determinar la apertura/encubrimiento de la orientación sexual de estudiantes pertenecientes al grupo de lesbianas, gais, bisexuales y otras minorías sexuales (LGB+) de la carrera de Medicina (campus de Sajonia y de San Lorenzo, y Filial Santa Rosa del Aguaray) de la Facultad de Ciencias Médicas de la Universidad Nacional de Asunción. El conocimiento de esta situación en el ámbito local permitirá adecuar intervenciones que aborden las preocupaciones sobre la apertura o el encubrimiento de la orientación sexual, discriminación de cualquier índole y la necesidad de apoyo emocional de manera a preservar y promover la salud mental de estos estudiantes (11).

\section{METODOLOGÍA}

Diseño

Este fue un estudio observacional descriptivo, de corte transversal y temporalmente prospectivo.

\section{Muestreo}

El muestreo fue no probabilístico, a criterio. Una encuesta en línea fue diseñada a través de Google Forms. Los investigadores compartieron el enlace de la encuesta en redes sociales (Facebook, Twitter, 
Instagram) y aplicaciones de mensajería (WhatsApp, Telegram), utilizadas por los estudiantes de la carrera de Medicina (campus de Sajonia y de San Lorenzo, y Filial Santa Rosa del Aguaray). Al inicio de la encuesta en línea, a los participantes se les pidió que confirmen que eran estudiantes de Medicina de la Universidad Nacional de Asunción y que voluntariamente aceptaban participar de la investigación.

\section{Instrumento}

El instrumento en línea de recolección de datos permitió examinar diversas variables. A los participantes se les consultó acerca de su edad (en años cumplidos), sexo (hombre, mujer, prefiere no decirlo), campus (Central [Sajonia o San Lorenzo], Filial Santa Rosa del Aguaray), curso $\left(1^{\circ}\right.$ al $\left.6^{\circ}\right)$, y sobre su orientación sexual (heterosexual, homosexual, bisexual, otras). A los participantes se les pidió que respondan preguntas acerca del grado de apertura/ocultamiento de su orientación sexual en distintos ámbitos. Para ello, se utilizaron adaptaciones propias en español de las escalas "Outness Inventory" y "Sexual Orientation Concealment Scale", previamente validadas en idioma inglés.

Outness Inventory: Desarrollado por Mohr \& Fassinger (11), es un inventario de 11 ítems diseñado para evaluar el grado en que las personas lesbianas, gais y bisexuales son abiertas sobre su orientación sexual. Las respuestas a los ítems del inventario indican el grado en que la orientación sexual del entrevistado es conocida y discutida abiertamente con varios tipos de individuos (por ejemplo, familia, compañeros de trabajo). Cabe destacar que, analizada la fiabilidad de la adaptación del inventario, este mostró una buena consistencia interna ( $\alpha$ de Cronbach $=0,90$ ).

Sexual Orientation Concealment Scale: Desarrollada por Jackson \& Mohr (12), esta escala consta de 6 ítems que permiten evaluar el ocultamiento activo que hacen las personas lesbianas, gais y bisexuales de su orientación sexual. La escala fue diseñada con base en elementos que reflejan el uso de estrategias de evitación (es decir, evitar circunstancias que implicarían a uno ser visto como perteneciente a una minoría sexual) y estrategias de fabulación (es decir, construir una apariencia falsa de heterosexualidad). Al promediar los seis elementos de la escala, se obtuvo la puntuación general de la misma. Aquí cabe destacar también que, analizada la fiabilidad de la versión española de la escala, la misma mostró una buena consistencia interna ( $\alpha$ de Cronbach=0,90).

Análisis estadístico

El tamaño de muestra fue calculado usando el paquete epidemiológico Epidat (Organización Panamericana de la Salud, Junta de Salud de Galicia y Universidad CES de Colombia). Asumiendo una frecuencia de orientación sexual distinta a la heterosexual del $15,8 \%$ en estudiantes de Medicina (3), un nivel de confianza del $95 \%$ y una precisión del $5 \%$, la muestra mínima quedó establecida en 205 participantes (13). Finalmente, la muestra incluyó a 277 estudiantes.

Los datos fueron cargados en una planilla de cálculo, para luego ser procesados con el paquete estadístico Epilnfo, versión 7.2. Las variables categóricas fueron resumidas en forma de tablas y las numéricas con medidas de tendencia central y dispersión. Para buscar asociaciones entre variables categóricas se utilizaron la prueba chi cuadrado de Pearson y el test exacto de Fisher, según sea el caso, considerando $p<0,05$ como significativa.

\section{Asuntos éticos}

El estudio se enmarcó en la línea de investigación "Epidemiología y psicopatología de los trastornos mentales" (instalada por Resolución D. N 1950/2017 del Decano de la Facultad de Ciencias Médicas de la Universidad Nacional de Asunción) y fue aprobado por la Cátedra de Psiquiatría de la Facultad de Ciencias Médicas de la Universidad Nacional de Asunción, Paraguay. En todo momento se respetaron los principios de ética aplicados a la investigación y la participación fue libre y voluntaria. Se respetaron los principios de autonomía, beneficencia y no maleficencia y de justicia, de acuerdo a la Declaración de Helsinki. En todo momento se respetó el anonimato de los participantes, pues no se recabaron datos que pudieran permitir la identificación de los mismos.

\section{RESULTADOS}

277 estudiantes de Medicina participaron de la investigación. 107 (38,62\%) fueron hombres, 166 $(59,92 \%)$ mujeres y 4 (1,46\%) prefirieron no decirlo. La media de edad fue de $23.3 \pm 2,1$ años. En cuanto a la orientación sexual, 229 participantes $(82,7 \%)$ se identificaron como heterosexuales y $48(17,3 \%)$ como parte de las minorías sexuales (lesbianas, gais, bisexuales, entre otras). Los datos sociodemográficos pueden verse en detalle en la tabla 2. 
TABLA 2. ORIENTACIÓN SEXUAL SEGÚN SEXO, SEDE Y CURSO DE LOS ESTUDIANTES DE MEDICINA DE LA UNIVERSIDAD NACIONAL DE ASUNCIÓN (N=277).

\begin{tabular}{|c|c|c|c|c|c|c|}
\hline & \multirow{3}{*}{ Características } & \multicolumn{4}{|c|}{ Orientación Sexual } & \multirow{3}{*}{$p$} \\
\hline & & \multicolumn{2}{|c|}{ Heterosexual } & \multicolumn{2}{|c|}{ LGB+ } & \\
\hline & & $\mathrm{n}$ & $\%$ & $\mathrm{n}$ & $\%$ & \\
\hline \multirow{3}{*}{ Sexo } & Hombre & 79 & 73,8 & 28 & 26,2 & $<0,01^{a}$ \\
\hline & Mujer & 149 & 89,8 & 17 & 10,2 & \\
\hline & Prefiere no decirlo & 1 & 25,0 & 3 & 75,0 & \\
\hline \multirow{2}{*}{ Sede } & Central & 215 & 83,0 & 44 & 17,0 & $0,527^{b}$ \\
\hline & Santa Rosa & 14 & 77,8 & 4 & 22,2 & \\
\hline \multirow{6}{*}{ Curso } & Primer curso & 25 & 71,4 & 10 & 28,6 & $0,146^{a}$ \\
\hline & Segundo curso & 51 & 87,9 & 7 & 12,1 & \\
\hline & Tercer curso & 12 & 80,0 & 3 & 20,0 & \\
\hline & Cuarto curso & 30 & 75,0 & 10 & 25,0 & \\
\hline & Quinto curso & 76 & 88,4 & 10 & 11,6 & \\
\hline & Sexto curso & 35 & 81,4 & 8 & 18,6 & \\
\hline
\end{tabular}

${ }^{a}$ Chi cuadrado de Pearson (considerando solo hombre y mujer).

${ }^{b}$ Test exacto de Fisher.

De los 48 participantes que se identificaron como parte de las minorías sexuales, $17(35,4 \%)$ refirieron ser homosexuales, 27 (56,3 \%) bisexuales, 1 (2 \%) mencionó ser heteroflexible, 1 (2\%) no definido y 2 participantes $(4,2 \%)$ se describieron como pansexuales. Se encontraron diferencias estadísticamente significativas en cuanto a los niveles de apertura de la orientación sexual en personas heterosexuales y personas $L G B+[t(275)=8,2, p<0,01]$. En la población de estudiantes de Medicina LGB+, se observaron menores niveles de apertura primero hacia la religión, y luego hacia la familia, Facultad y amigos $(p<0,01)$ (gráfico 1). Tomando en cuenta las distintas identidades que forman el espectro LGB+, el menor nivel de apertura fue aquel relacionado con la religión, en todos los casos (gráfico 2).

\section{GRÁFICO 1. NIVELES DE APERTURA DE LA ORIENTACIÓN SEXUAL DE ESTUDIANTES DE MEDICINA HETEROSEXUALES VS ESTUDIANTES DE MEDICINA LGB+. RESULTADOS DEL OUTNESS INVENTORY ( $\mathrm{N=277).}$}

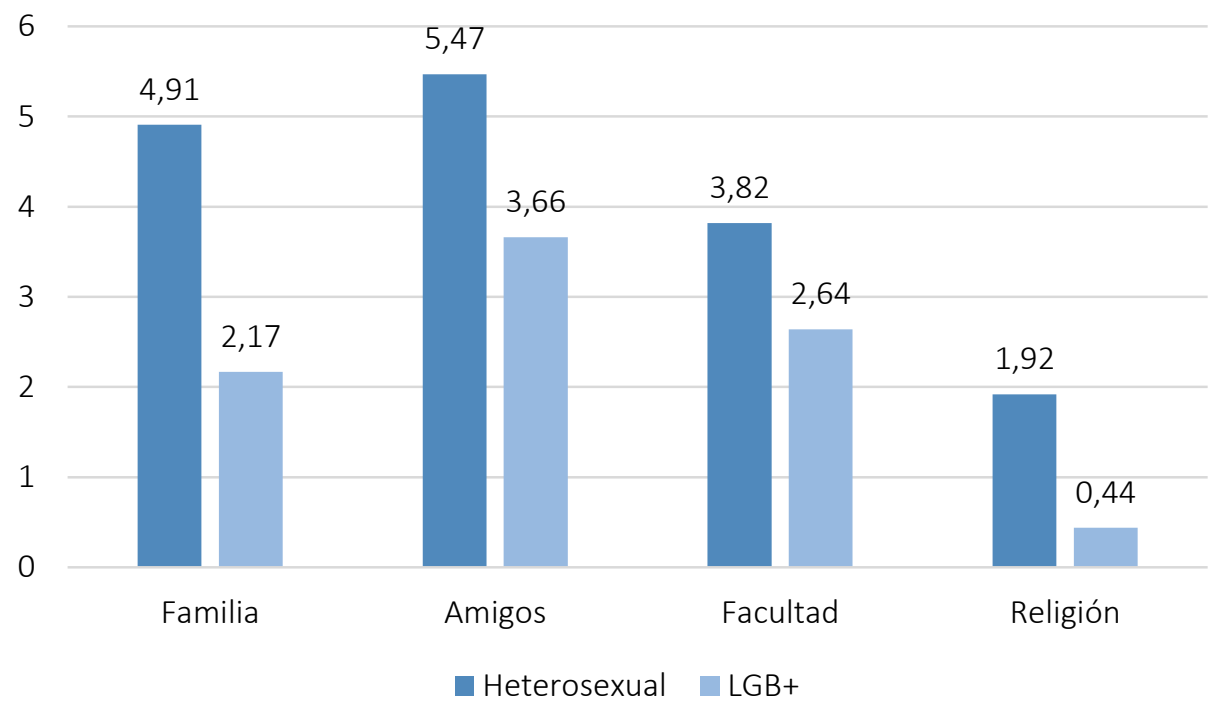


GRÁFICO 2. NIVELES DE APERTURA DE LA ORIENTACIÓN SEXUAL DE ESTUDIANTES DE MEDICINA LGB+. RESULTADOS DEL OUTNESS INVENTORY ( $\mathrm{N}=\mathbf{2 7 7})$.

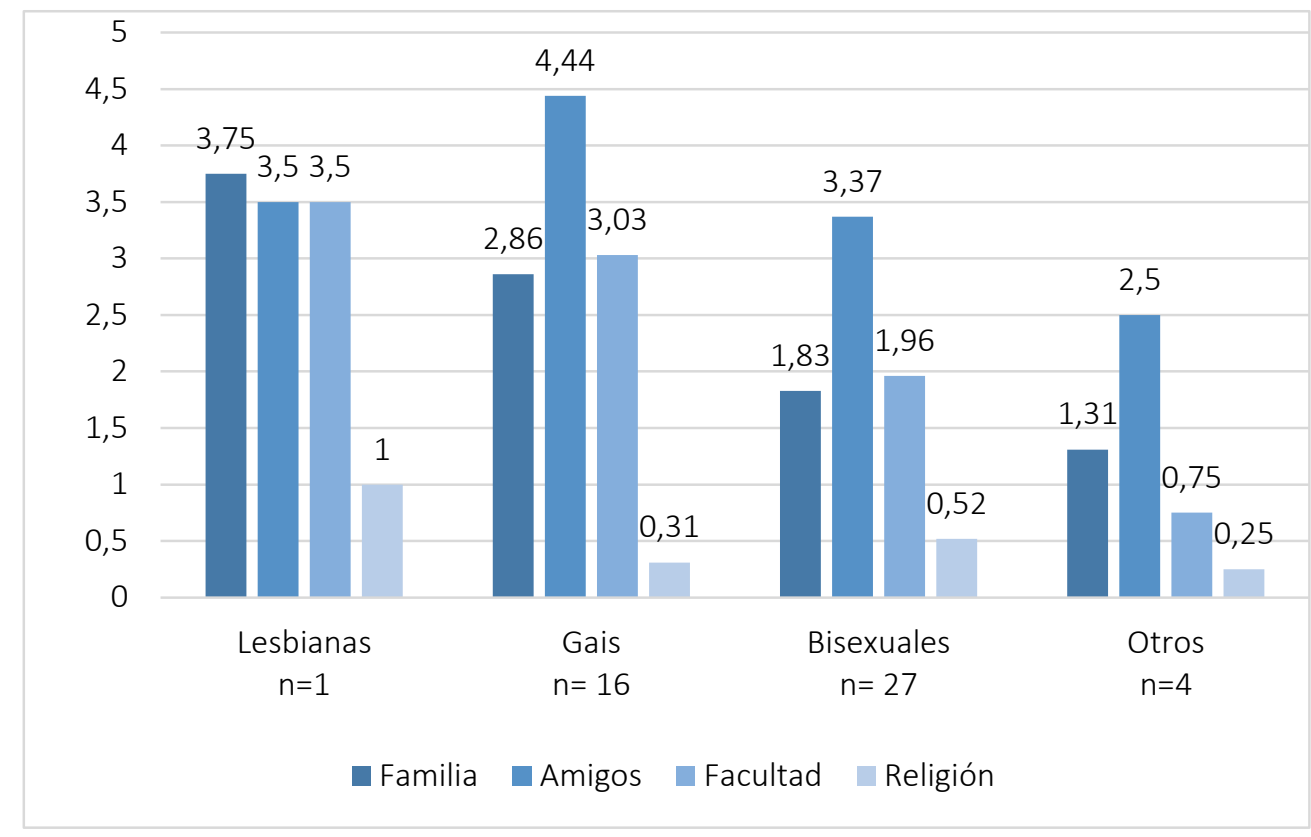

36 (75 \%) de los 48 participantes del grupo LGB+ ocultaron de alguna forma su orientación sexual en las últimas dos semanas, y el promedio grupal obtenido en la Sexual Orientation Concealment Scale fue de 0,90.

Específicamente, 11 (23\%) ocultaron su orientación sexual afirmando que eran heterosexuales o negando que eran LGB+. Además, 9 participantes (18,75 \%) lo hicieron evitando el contacto con otros individuos LGB+, y 14 (29,2 \%) evitaron temas de sexo, amor, atracción o relaciones. Asimismo, 31 encuestados (65 \%) permitieron que los demás asumieran que eran heterosexuales, sin corregirlos. Finalmente, 12 (25\%) alteraron su apariencia, gestos o actividades para "pasar" de heterosexuales y 16 (33,3 \%) permanecieron en silencio mientras presenciaban comentarios, chistes o actividades homofóbicas por miedo a que se les etiquetara como LGB+.

\section{DISCUSIÓN}

Salir del clóset en un ambiente educativo puede llegar a ser muy desafiante. Por ejemplo, un estudio realizado en España (14) investigó la experiencia escolar en la narrativa de la orientación sexual LGB+, llegando a la conclusión de que había una falta generalizada de herramientas para personas de este grupo a la hora de salir del clóset, lo que convertía su experiencia en una situación abrumadora y hasta paralizante. Si bien nuestro estudio fue realizado en población universitaria, y a pesar de las obvias diferencias etarias, las personas del grupo LGB+ de la presente investigación (el 17,3 \% de la población estudiada) también carecen de esas narrativas, lo que se refleja en la necesidad de ocultar su orientación sexual en el ambiente de la Facultad de Ciencias Médicas de la Universidad Nacional de Asunción. Lo anterior podría deberse a la falta de herramientas ofrecidas por la Facultad de Ciencias Médicas para que sus estudiantes expresen libremente su orientación sexual, por lo que los estudiantes LGB+ deben inhibir comportamientos considerados como no heterosexuales.

Lo anterior está en consonancia con una reciente revisión sistemática (15) que exploró factores de riesgo y protección relacionados con la homofobia interiorizada. Este tipo de homofobia se define como el estado en el que las personas LGB+ valoran negativamente su orientación sexual, identidad, apariencia y expresión de género que no se ajusta al modelo binario heteronormativo que predomina en la sociedad (16), tal como se ha visto en los resultados de nuestra investigación. Esta percepción negativa puede ser dirigida tanto hacia el interior de la persona (expresándose en forma de rechazo a la propia orientación sexual, experimentando sentimientos de culpa y vergüenza al comentar su orientación sexual a otras personas o al mantener relaciones sexuales con personas de su mismo sexo) como hacia su exterior (manifestándose como rechazo hacia otras personas del colectivo LGB+, o criticando diferentes expresiones de género asociadas a la homosexualidad) (17).

Un estudio realizado en Nueva York (18) investigó acerca de la apertura de la orientación sexual en estudiantes de Medicina y médicos postulantes a una residencia médica, todos pertenecientes a la comunidad LGB+. El $95 \%$ de todos los encuestados decidió no revelar su orientación sexual al momento de 
ser admitidos a la escuela de Medicina. De esos, el 17 \% refirió sentirse incómodo al revelar esa información, y el $15 \%$ pensaba que no iban a ser admitidos como estudiantes de Medicina si lo hacían. Además, durante el proceso de selección de una residencia médica, el 52 $\%$ de los encuestados no estaba seguro de revelar su orientación sexual. De esos, el 33 \% mencionó que lo decidirían una vez que conozcan mejor el ambiente del programa de residencia. De aquellos que no planeaban revelar su orientación sexual, más de la mitad afirmó que no lo haría porque les preocupa no ser aceptados. La aceptación de ser de la comunidad LGB+ (ya sea por el ambiente del programa de residencia, por sus compañeros, o supervisores) es siempre un tema destacado a la hora de decidir revelar u ocultar la orientación sexual en las minorías sexuales (18).

Otro estudio estadounidense, que incluyó a 1334 estudiantes de Medicina, concluyó que los estudiantes LGB+ se sentían más incómodos $(z=-9,0 ; p<, 000)$ con revelar su orientación sexual que los estudiantes heterosexuales (19). Esto coincide con nuestra investigación, que encontró diferencias estadísticamente significativas en cuanto a los niveles de apertura de la orientación sexual en personas heterosexuales y en personas LGB+ $[\mathrm{t}(275)=8,2$, $p<0,01]$. Asimismo, el estudio antedicho encontró que aquellos estudiantes con niveles más altos de incomodidad a la hora de revelar su orientación sexual también tenían niveles más altos de depresión.

El nivel de apertura de la orientación sexual interacciona e impacta en la salud mental de los individuos. Investigadores han demostrado que un mayor grado de apertura sobre la orientación sexual se ha relacionado con una mejor salud mental en general, incluso luego de controlar factores de confusión como la edad, raza y sexo (20). No obstante, la apertura tiene sus desafíos: muchas veces las personas LGB+ que están fuera del clóset están expuestas al prejuicio y a la discriminación, factores que definitivamente pueden tener un impacto negativo en la salud mental (21). Por lo tanto, el nivel de apertura de la orientación sexual puede tener consecuencias positivas o negativas para la salud mental, por lo que más investigación es necesaria para poder dilucidar esa naturaleza dual y multifacética. Nuestra investigación podría servir para dar el puntapié inicial para la realización de más estudios locales que permitan entender cómo la apertura sobre la orientación sexual afecta al bienestar emocional, psicológico y social de las minorías sexuales, específicamente de estudiantes de Medicina LGB+.

Investigadores mexicanos (22) estudiaron las actitudes de estudiantes universitarios hacia sus compañeros y profesores de la comunidad LGB+, encontrando una frecuencia de actitudes desfavorables del 24,9\% (siendo los hombres aquellos que presentaban la mayor proporción de actitudes desfavorables). Justamente, muchos estudiantes de Medicina LGB+ informan que prefieren ocultar su orientación sexual debido al miedo a las reacciones y actitudes negativas de sus compañeros y profesores (23). Con matices diferentes, un estudio realizado en Paraguay, específicamente en la Filial Santa Rosa del Aguaray de la Facultad de Ciencias Médicas de la Universidad Nacional de Asunción, examinó la actitud de los estudiantes de Medicina hacia la homosexualidad en general (24). El 42,9 \% de los participantes se mostró indiferente 0 indeciso en su actitud hacia la homosexualidad y $28,6 \%$ tuvo actitudes discriminatorias. La actitud se define como la suma de sentimientos, prejuicios, ideas, miedos o creencias que tiene un individuo sobre un tema específico. Su expresión es la opinión de aceptación o rechazo por parte de una persona sobre una condición particular. La actitud también puede considerarse un conjunto de creencias, sentimientos y tendencias que incluyen tres componentes: cognitivo, afectivo y conductual (25-27).

Las actitudes negativas de los estudiantes de Medicina hacia las personas LGB+ pueden hacer que las mismas se sientan maltratadas, discriminadas y disuadirlas de buscar atención médica. Por ello, es importante estudiar la actitud de los estudiantes de Medicina hacia la homosexualidad, porque el prejuicio podría dañar la calidad de la atención médica de los pacientes LGB+y, naturalmente, afectar a compañeros/profesores que también se identifican como parte de las minorías sexuales $(28,29)$.

Finalizando, en esta investigación se evidenciaron diferencias estadísticamente significativas en cuanto a los niveles de apertura de la orientación sexual en personas heterosexuales $y$ en personas LGB+. Específicamente, la población de estudiantes de Medicina LGB+ mostró menores niveles de apertura primero hacia la religión, y luego hacia la familia, Facultad y amigos. Asimismo, la mayoría de los encuestados ocultó de alguna forma su orientación sexual, lo que sugiere la existencia de homofobia interiorizada. Todo esto es importante, puesto que estudios previos han indicado que la apertura y aceptación sobre la orientación sexual son importantes elementos para los estudiantes de Medicina LGB+ (3034).

Con base en estos resultados, la Facultad de Ciencias Médicas de la Universidad Nacional de Asunción debe considerar examinar de cerca su ambiente y cultura organizacional, así como su currículo, con el fin de 
garantizar que exista un entorno positivo e inclusivo para todos los estudiantes. La adopción de la diversidad en la educación médica permite formar médicos que puedan practicar la Medicina de manera competente, compasiva y con enfoque de derechos humanos. Asimismo, se necesita más educación y formación sobre los problemas de salud de las personas LGB+. La actitud hacia la homosexualidad es un tema importante en la educación médica, pues puede afectar la calidad de la atención de pacientes $\mathrm{LGB}+$ y distorsionar la relación médico-paciente.

Una limitación del presente estudio la constituye el tipo de muestreo utilizado, puesto que, al ser un muestreo de tipo no probabilístico, no pueden generalizarse sus resultados. Otra limitación fue el hecho de no haber incluido a estudiantes de las demás carreras con que cuenta la institución (Kinesiología y Fisioterapia, Instrumentación y Área Quirúrgica, y Podología). Estos estudiantes podrían experimentar situaciones distintas respecto a la apertura/encubrimiento de su orientación sexual. Todo esto debe ser tenido en cuenta en futuros estudios, con el fin de establecer cuáles son las áreas que se necesitan intervenir para disminuir los niveles de homofobia internalizada y favorecer la libertad de expresión de los estudiantes de Medicina LGB+. Otra limitación es que no se exploraron las motivaciones o factores que inciden en la decisión de los estudiantes de Medicina LGB+ de la Universidad Nacional de Asunción de salir o mantenerse dentro del clóset. Una investigación de diseño cualitativo sería de utilidad para explorar esta área y aumentar nuestra comprensión sobre la misma.

\section{AGRADECIMIENTOS}

Los autores agradecen a Fabián Forestieri y a Augusto Marecos, miembros titulares de la Junta Directiva Nacional de Amnistía Internacional Paraguay, por sus aportes al constructo teórico de esta investigación.

\section{REFERENCIAS}

1. Tetreault PA, Fette R, Meidlinger PC, Hope D. Perceptions of campus climate by sexual minorities. J Homosex. 2013;60(7):947-964.

https://doi.org10.1080/00918369.2013.774874

2. American Psychological Association. Orientación sexual e identidad de género [Internet]. American Psychological Association. 2020 [citado el 29 de octubre de 2020]. Disponible

en: https://www.apa.org/centrodeapoyo/sexual

3. Mansh M, White W, Gee-Tong L, Lunn MR, Obedin-Maliver $J$, Stewart L, et al. Sexual and gender minority identity disclosure during undergraduate medical education: "in the closet" in medical school. Acad Med. 2015;90(5):634-644. https://doi.org/10.1097/ACM.0000000000000657

4. Dianderas Wong DA. El proceso de aceptación de una identidad sexual homosexual en hombres jóvenes de lima [tesis]. Lima: Pontificia Universidad Católica del Perú; 2015. URL.

5. Lird M, Barrios I, Torales J. Discriminación en el ambiente laboral hacia personas con orientación sexual diferente a la heterosexual: un estudio en funcionarios públicos de la Junta Municipal de la ciudad de Asunción, Paraguay. Medicina Clínica y Social. 2018;2(2):84-93. URL.

6. Diccionario de la lengua española. Fobia [Internet]. $24^{\mathrm{a}}$ ed. Madrid: RAE; 2014 [citado el 29 de octubre de 2020]. Disponible en: https://dle.rae.es/fobia

7. Tin LG. Diccionario de la homofobia [Internet]. Tres Cantos: Akal; 2012 [citado 27 de septiembre de 2020]. URL. Disponible en: https://www.akal.com/libro/diccionario-dela-homofobia 34589/

8. Cruz Sierra S. Homofobia y masculinidad. Cotidiano/México. 2002;18(113):8-14. URL.

9. Zamani-Gallaher EM, Choudhuri DD. A primer on LGBTQ students at community colleges: considerations for research and practice. New Dir Community Coll. 2011;155:35-49. https://doi.org/10.1002/cc.456

10. British Medical Association Equal Opportunities Committee. Career Barriers in Medicine: Doctors' Experiences [Internet]. British Medical Association Equal Opportunities Committee. 2004 [citado el 27 de octubre de 2020]. Disponible en: http://www.hscbusiness.hscni.net/pdf/BMACareer Barriers in Medicine-2004 pdf.pdf

11. Mohr J, Fassinger R. Measuring dimensions of lesbian and gay male experience. Meas Eval Couns Dev. 2000;33(2):6690. https://doi.org/10.1080/07481756.2000.12068999

12. Jackson SD, Mohr JJ. Conceptualizing the closet: Differentiating stigma concealment and nondisclosure processes. Psychol Sex Orientat Gend Divers. 2016;3(1):8092. https://doi.org/10.1037/SGD0000147

13. Muñoz Navarro SR. ¿Cuántos sujetos necesito para mi estudio? Medwave. 2014;14(6):e5995. https://doi.org/10.5867/medwave.2014.06.5995

14. Ramírez Pavelic M. La experiencia en las narrativas de identidad sexual LGTB: un estudio fenomenológico retrospectivo [tesis]. Madrid: Programa de Psicología Evolutiva y de la Educación, Universidad Autónoma de Madrid, Facultad de Psicología; 2012. URL.

15. Castellano Melguizo A. Homofobia interiorizada: factores de riesgo y de protección. Una revisión sistemática de la literatura. Jaén: Universidad de Jaén; 2020. Disponible en: https://tauja.ujaen.es/jspui/handle/10953.1/10658

16. Mohr JJ, Kendra MS. Revision and extension of a multidimensional measure of sexual minority identity: the Lesbian, Gay, and Bisexual Identity Scale. J Couns Psychol. 2011;58(2):234-245. https://doi.org/10.1037/a0022858

17. Ludici A, Verdecchia M. Homophobic labeling in the process of identify construction. Sexuality \& Culture 2015;19: 737-758. https://doi.org/10.1007/s12119-015$\underline{9287-0}$

18. Merchant RC, Jongco AM 3rd, Woodward L. Disclosure of sexual orientation by medical students and residency applicants. Acad Med. 2005;80(8):786. https://doi.org/10.1097/00001888-200508000-00017

19. Lapinski J, Sexton P. Still in the closet: the invisible minority in medical education. BMC Med Educ. 2014;14:171. https://doi.org/10.1186/1472-6920-14-171

20. Feldman SE, Wright AJ. Dual Impact: Outness and LGB Identity Formation on Mental Health. J Gay Lesbian Soc Serv. 2013;25(4):443-464.

https://doi.org/10.1080/10538720.2013.833066

21. Benton J. Making schools safer and healthier for lesbian, 
gay, bisexual, and questioning students. J Sch Nurs. 2003;19(5):251-259.

https://doi.org/10.1177/10598405030190050201

22. Barragán V. Actitudes de estudiantes universitarios hacia sus compañeros de clase y profesores de la comunidad lésbico-gay [tesis doctoral]. Ciudad de México: Programa de Maestría en Ciencias Médicas, Odontológicas y de la Salud, Universidad Nacional Autónoma de México; 2011. URL.

23. Risdon C, Cook D, Willms D. Gay and lesbian physicians in training: a qualitative study. CMAJ. 2000;162(3):331-334. URL.

24. Torales J, Barrios I, Torres A, Dunjo N, Benitez MG, Villalba $\mathrm{J}$, et al. Attitude of Medical Students in Paraguay Towards Homosexuality. East Asian Arch Psychiatry. 2018;28(3):101103. URL.

25. Soto-Cáceres V. Actitudes sobre el aborto, matrimonio homosexual y otros aspectos socio sexuales en estudiantes del sexto año de medicina humana en una universidad estatal. Rev. Cuerpo Méd. Hosp. Nac. Almanzor Aguinaga Asenjo 2014;7(2):6-10. URL.

26. Summers G. Medición de Actitudes. Ciudad de México: Editorial Trillas; 1979.

27. Sueiro Domínguez E, Diéguez Ruibal JL, López Sánchez F, López Castedo A. Propiedades psicométricas de la escala de actitudes hacia la sexualidad (ATSS) ampliada. Cuadernos de medicina psicosomática y psiquiatría de enlace 2005;74:46-56. URL.

28. Campo-Arias A, Oviedo HC, Herazo E. Correlación entre homofobia y racismo en estudiantes de medicina.
Psicología desde el Caribe 2014;31(1):25-37. https://doi.org/10.14482/psdc.31.1.5331

29. Costa PA, Almeida R, Anselmo C, Ferreira A, Pereira H, Leal I. University students' attitudes toward same-sex parenting and gay and lesbian rights in Portugal. J Homosex. 2014;61(12):1667-1686.

https://doi.org/10.1080/00918369.2014.951253

30. Oriel KA, Madlon-Kay DJ, Govaker D, Mersy DJ. Gay and lesbian physicians in training: family practice program directors' attitudes and students' perceptions of bias. Fam Med. 1996 Nov-Dec;28(10):720-725. URL.

31. Townsend MH, Wallick MM, Cambre KM. Support services for homosexual students at U.S. medical schools. Acad Med. 1991;66(6):361-363. https://doi.org/10.1097/00001888-199106000-00012

32. Townsend $\mathrm{MH}$, Wallick MM, Cambre KM. Follow-up survey of support services for lesbian, gay, and bisexual medical students. Acad Med. 1996;71(9):1012-1014. https://doi.org/10.1097/00001888-199609000-00018

33. Stott DB. The training needs of general practitioners in the exploration of sexual health matters and providing sexual healthcare to lesbian, gay and bisexual patients. Med Teach. 2013;35(9):752-759. https://doi.org/10.3109/0142159X.2013.801943

34. Sitkin NA, Pachankis JE. Specialty Choice Among Sexual and Gender Minorities in Medicine: The Role of Specialty Prestige, Perceived Inclusion, and Medical School Climate. LGBT Health. 2016;3(6):451-460. https://doi.org/10.1089//gbt.2016.0058 